\title{
Italienische Musiker am Hof des Emmerich Esterházy in Preßburg und die Kontakte mit der Kaiserlichen Hofmusikkapelle in Wien
}

\section{Italian Musicians at the Court of Emericus Esterházy in Preßburg and Contacts with the Imperial Court Ensemble in Vienna}

Ladislav Kačic / ladislav.kacic@savba.sk

Jan Stanislav Institute of Slavistics of the Slovak Academy of Sciences, Bratislava, SK

\begin{abstract}
Archbishop-primate Emericus Esterházy (1663-1745) belonged to the greatest patrons of the arts in the history of Bratislava. His ensemble consisted of many prominent musicians, where Italians held a significant role. Bassoonist Angelo Cavallari was the first Italian member of the Esterházy ensemble, working there in the years 1730-1734. Before, he had applied for an employment in the Imperial Court Ensemble (Kaiserliche Hofmusikkapelle) in Vienna. In 1737, well know violinist Giacomo Calandro (1707-1788) came to Preßburg and 1740, even renowned Francesco Durante (1684-1755) was there. However, he stayed in Preßburg only for a year and subsequently he returned to Naples. In the same year as Durante, castratos Domenico Tasselli and Dario Luca Catani also came to the Esterházy court. Previously, they had worked in Moravia, Tasselli also in Prague. At the end of 1743, also singer (contraalto) of Hofmusikkapelle Filippo Antonelli (1710-1760) joined the archbishop's ensemble. Antonelli was not the only musician who worked in Vienna and Preßburg at the same time. In 1731 already, violinist of Imperial Court Ensemble Johann Otto Rossetter (ca. 1680-1754) with his son Johann Killian (1710-?) began to serve for the Esterházy court. E. Esterházy contacts with the Imperial Court in Vienna were very intense. He send his musicians there for the further studies (for example, 1734 the prospective Kapellmeister Joseph Umstatt who was sent to J. J. Fux). Court instrument makers (Antoni Posch, Michael Leichamschneider) often worked for the Esterházy court as well.
\end{abstract}

\section{Keywords}

Bratislava (Preßburg), Archbishop E. Esterházy, Italians, F. Durante, castratos, Imperial Court Ensemble, Vienna 
Der Fürstbischof und Primas von Königreich Ungarn Emmerich Esterházy (1663-1745) ${ }^{1}$ gehörte zu den größten Mäzenen der Kunst in der Geschichte der Slowakei. An seinem Hof in Preßburg wirkten z. B. solche bildende Künstler wie G. R. Donner und A. Galli Bibiena. ${ }^{2}$ Bis vor kurzem wusste man nicht, dass Esterházy auch eine ausgezeichnete Kapelle hatte. An seinem Hof wirkten in den Jahren 1725-1745 ca. 50 Musiker - Österreicher, Deutsche, Italiener, Franzosen, Musiker aus Böhmen und Mähren und natürlich auch einheimische Musiker, d. h. Slowaken (die Liste der Musiker siehe in der Beilage). ${ }^{3}$ Es war also eine typische internationale adelige Kapelle des 18. Jahrhunderts. Zu den besten Musikern am Hofe Esterházys gehörten bestimmt immer die Italiener.

Der erste italienische Musiker bei Esterházy war der Fagottist Angelo Cavallari. Er stammte aus Verona und kam nicht direkt aus Italien nach Preßburg, sondern aus Wien. Im Jahr 1727 hat er sich nämlich um die Stelle des Fagottisten der Kaiserlichen Hofmusikkapelle in Wien beworben (siehe Abbildung 1). ${ }^{4}$ Das beweist das eigenhändig geschriebene Dokument, wobei Cavallari schreibt, dass er bei einer Opernaufführung in der Favorita mitgespielt hat. Es war die am 30. 8. 1727 zum Namenstag der Kaiserin Elisabeth Christina aufgeführte Oper Imeneo A. Caldaras. Cavallari hatte aber Pech gehabt. Der Hofkapellmeister Fux hat ihn „weder gelobt, noch verachtet“, wobei aber das wichtigste Argument für die negative Stellungnahme die volle Besetzung der Fagottisten-Stellen in der Kaiserlichen Hofmusikkapelle war: neben dem „alten“ Carl Maillard „noch 4 andere gute Fagottisten vorhanden seynd“. ${ }^{5}$

Deshalb ist Cavallari im Juli 1730 in die Dienste des E. Esterházy in Preßburg getreten. Zum ersten Mal hat er seinen Lohn (79 Fl 30 Xr) - zusammen mit dem späteren Kapellmeister („Music-Director“) Joseph Umstatt ${ }^{6}$ - für das 3. Quartal 1730 bekommen.

1 Mehr über E. Esterházy (Literatur usw.) siehe z. B. SCHMITTH, P. Nicolaus SJ. Archiepiscopi Strigonienes. Pars secunda. Tyrnavie: Typis Acemicis Societatis Jesu 1758, S. 194-217; BAHLCKE, Joachim. Ungarischer Episkopat und österreichische Monarchie. Stuttgart: Franz Steiner Verlag 2005, S. 132-135; KOLTAI, András. Esterházy Imre. In Esztergomi ersékek. Ed. Márgit Beke. Budapest: Szent Istán társulat 2003, S. 331-331; KOLTAI, András. Császárhű bökezű remete, Esterházy Imre herczegprímás. In Limes 2005, S. 375-418; FORGÓ, András. Esterházy Imre és az aulikus politika a 18. század első évtizedelben. In „Fényes palotákban, édes köfalokban“ (Tanulmányok az Eszterházy családról a források tükrében). Budapest 2009, S. 65-86.

2 Vgl. PÖTZL-MALÍKOVÁ, Mária. Juraj Rafael Donner a Bratislava. Bratislava: Tatran 1993; GALAVICS, Géza. Antonio Galli Bibiena in Ungheria e Austria. Acta Historiae Artium, Jg. 30, 1984, S. 177-263.

3 Die erste Studie über die Esterházysche Kapelle siehe KAČIC, Ladislav. Kapela Imricha Esterházyho v rokoch 1725-1745. Musicologica slovaca, 5 (31), 2014, S. 189-254.

4 ÁGUSTSSON, Jóhannes. "La perfetta cognitione”. Francis Stephen of Lorraine, Patron of Vivaldi. Studi vivaldiani 15 (2015), S. 145. Ich danke meinem Kollegen J. Águstsson für die freundliche Übersendung der digitalen Kopie des Dokumenten herzlichst.

5 Tatsächlich waren es Franz Sturm, Tobias Woschitka, Johann Jacob Friedrich und Anton Maillard. Vgl. KÖCHEL, Ludwig Ritter von. Die Kaiserliche Hofmusikkapelle in Wien von 1543 bis 1867. Wien: Becksche Universitätsbuchhandlung 1869, S. 79.

6 Mehr über Joseph Umstatt siehe KAČIC, Ladislav. Joseph Umstatt (1711-1762) zwischen Barock und Klassik. Bemerkungen zur Stilentwicklung eines mitteleuropäischen Komponisten. In (Ed. Gerold Gruber): Zur Geschichte und Aufführungspraxis der Musik des 16.-18. Jahrhunderts in Mittel- und Osteuropa. Bratislava: Music- 
Cavallari wohnte in Preßburg nicht im Primatialpalais, sondern bei einem anderen Hofbeamten - bei dem Tapezierer F. Pulsatori, wofür er weitere $6 \mathrm{Fl}$ per Quartal bekommen hat. Am 30. 12. 1730 hat er 40,- Fl „pro vestitu“ erhalten, es war nach dem Kaiserlichen Geiger J. O. Rossetter (50,- Fl) die zweithöchste Summe an Kleidergeld.

Cavallari stammte wahrscheinlich aus einer verzweigten Musikerfamilie: Giacomo Cavallari (gest. 1753) war Vizekonzertmeister in Bayreuth, Francesco Cavallari war 17561787 Flötist der Hofkapelle in München, der aus Venedig stammende Oboist Giordano Cavallari wiederum 1720-1732 am Hof von Johann Adam Liechtenstein in Wien. ${ }^{7}$ Angelo Cavallari müsste ein guter Fagottist gewesen sein, trotzdem spielte er auch die Traversflöte, weil ihm am 2. 7. 1732 ein neues Instrument gekauft wurde („16 Fl per Flauto traverso“). Cavallari hat mehrmals selbst auch Blätter, oder S-Rohr („Fagottröhr“) in Wien angekauft (17.12.1733 - „4 Fl per anchie da fagotto riceuute da Vienna“; 8. 8. 1734 - „Fiorini quattro per anchie, ouuer canne di Fagotto fatte uenir da Vienna“). Im Oktober 1731 wurden für ihn in Wien 6 Fagottkonzerte gekauft: ${ }^{8}$

\section{Li 19 Gennaro 1732}

Certifico io sottoscritto auer riceuuto del Sig. ${ }^{r}$ Inspettore fiorini 12 per

sei concerti di fagotto comprati a Vienna nel mese d'ottobre dell' anno scadutto

Angelo Cauallari

Esterházy schätzte bestimmt A. Cavallari sehr hoch, weil dieser am Ende seines Wirkens in Preßburg am 10.11.1734 nicht nur seine volle Belohnung für das ganze 4. Quartal, sondern auch weitere 100 Florene für seine Rückreise nach Italien bekommen hat:

Io sottoscritto attesto d'auer ricevuto dal Molt‘ Ill ${ }^{r}$ Sig. Tereck [= Franz von Török, Expeditor am Hof] fiorini cento quali mi ha Sua Altezza Reuerendissima in gratia accordati, per il mio uiatico, per poter partir a casa mia in Italia. Dico -

$F 100=$

Angelo Cauallari

\footnotetext{
forum 2013, S. 201-215; WEINZIERL, Gerhard. Bamberger Hofmusik. Von der Gegenreformation bis zum Beginn des 19. Jahrhunderts. Würzburg: Königshausen und Neumann 2016, S. 168-193. KAČIC, Ladislav. Kapela Imricha Esterházyho (Anmerkung 3), S. 193, sowie UMSTATT, Joseph. Concerti per violino, bzw. Concerti per flauto traverso, hrsg. von Ladislav Kačic. Bratislava: Vysoká škola múzických umení 2013, bzw. 2017.

7 HEGEN, Irene. Die markgräfliche Hofkapelle zu Bayreuth (1661-1769). In LEOPOLD, Silke - PELKER, Bärbel (Hg.). Süddeutsche Hofkapellen im 18. Jahrhundert (online Publikation). Heidelberg: Heidelberger Akademie der Wissenschaften 2014, S. 32. MÜNSTER, Robert. Die Münchener Hofmusik bis 1800. Ebenda, S. 394. ÁGUSTSSON, Jóhannes. Joseph Johann Adam of Liechtenstein, Patron of Vivaldi. Studi Vivaldiani 17 (2017), S. 28-30, 42, 45-46, 48. Über die verwandschaftlichen Beziehungen der Cavallaris ist z. Z. nichts Konkretes bekannt.

8 Der Autor/Autoren ist/sind, leider, nicht angegeben. GERICKE, Hannelore. Wiener Musikalienhandel von 1700 bis 1778. Graz: Böhlau 1960, führt keine Fagottkonzerte an. Konzerte für Fagott hat jedoch sogar der Mitglied der Esterházyschen Kapelle Leopold Carl komponiert.
} 
Im April 1737 ist ein anderer guter italienischer Instrumentalist nach Preßburg gekommen - der Geiger Giacomo Calandro (Frasso Telesino 1707 - Neapel 1788). ${ }^{9}$ Er war der ältere Bruder des Komponisten und Kapellmeisters Nicola Calandro.

Giacomo Calandro studierte am Conservatorio dei Poveri di Gesù Cristo und Conservatorio della Pietà in Neapel und wirkte zuerst als Geiger in Neapel (Chiesa di Santa Maria) und dann in verschiedenen Operngesellschaften und -theatern in Italien, z. B. 1732 im Teatro Capranica in Rom. Am Hof Esterházys war Calandro damals (1737) der bestbezahlte Musiker: sein Jahreslohn war 300 Fl, vierteljährlich bekam er regelmäßig 111,- Fl. NB. besser bezahlt wurden nur zwei Ärzte (125 fl), sowie der Expeditor und Cassa-Verwalter F. von Török (162 Fl). Joseph Umstatt bekam z. B. 1738 als „Music-Director“ ein ähnliches Gehalt (111,- Fl) nur mit sog. „adjuta“ (73,30+37,30 Fl), der Konzertmeister L. Carl hatte z. B. (bis 1744) einen ständigen Lohn von 96,- Fl usw.

Calandro blieb in Preßburg bis Ende 1745, d. h. bis zum Tod des Fürstbischofs. Dann war er ab spätestens 1750 Konzertmeister der Operngesellschaft G. B. Locatellis in Prag. ${ }^{10}$ Calandro und Locatelli kannten sich aber bestimmt schon früher. Calandro war nämlich bei der Taufe des in Preßburg geborenen Sohns Locatellis und seiner Gemahlin, der Primadona Giovanna della Stella (Carlo Angelo Maria) am 3.2.1742 im Preßburger St.-Martins Dom anwesend (siehe Abbildung 2). Locatelli und seine Frau waren damals (seit 1741) als Mitglieder der Mingottischen Operngesellschaft, die während der Krönung Maria Theresias spielte, noch immer in Preßburg. ${ }^{11}$ Calandro, der dann eine gewisse Zeit bei Locatelli tätig war, später in Braunschweig und schließlich um 1770 in sein Heimatland zurückgekehrt ist, war bestimmt ein überdurchschnittlicher Geiger, wovon schon seine hohe Entlohnung ein klares Zeugnis ablegt.

Der beste Musiker am Hof des Fürstbischofs Emmerich Esterházy (d. h. nicht nur unter den Italienern, sondern überhaupt) war bestimmt Francesco Durante (Frattamaggiore 1684 - Neapel 1755). Er ist im Herbst 1739 von seiner Stelle als Lehrer und Kapellmeister am Conservatorio dei Poveri di Gesù Cristo in Neapel abgetreten. Darüber, wo er sich bis 1742 aufhielt, als er an einen ähnlichen Posten, allerdings am Conservatorio di Santa Maria di Loreto, nach Neapel zurückgekehrte, war bis vor kurzem nichts Konkretes bekannt (wo er lebte und wirkte). ${ }^{12}$ Vier überlieferte Quittungen über seine Quartalbelohung (siehe Abbildung 3) beweisen, dass er das ganze Jahr 1740 in Preßburg am Hof E. Esterházys lebte. Durante ist höchstwahrscheinlich über Wien nach Preßburg

9 Näheres über Giacomo Calandro siehe AMORE, Adriano. Giacomo Calandro, un violinista del '700. Le vie della musica, Jg. 8., 2007, Nr. 46, S. 38-39.

10 JONÁŠOVÁ, Milada. Italské operní árie na svatovítském kůru v Praze - Sehlingova éra. Hudebni věda, 38, 2001, S. 283. Über G. B. Locatelli siehe auch JAKUBCOVÁ, Alena und Kol. Starši divadlo v českých zemích do konce 18. stoleti (Osobnosti a díla). Praha: Divadelní ústav - Academia 2007, S. 350-354.

11 Vgl. MÜLLER, Erich H. Angelo und Pietro Mingotti. Ein Beitrag zur Geschichte der Oper im XVIII. Jahrhundert. Dresden: Richard Bertling Verlag, 1917, S. 21, XLIX.

12 Vgl. „Durante“ In Die Musik in Geschichte und Gegenwart, Kassel - Basel - London - Tours: Bärenreiter Verlag 1954, Sp. 986-993, sowie in The New Grove Dictionary of Music and Musicians, Vol. 7, London: Oxford University Press 2001, S. 739-745, sowie DIETZ, Hanns-Bertold. The Neapolitan School: Francesco Durante (1684-1755) - Aspects of Manuscript Dissemination, Misattributions, and Reception. Music en Perspectiva, Jg. 2, 2009, Nr. 2, S. 7-30. 
gekommen, weil er im Herbst 1739 in Wien einige Werke, u. a. Salve Regina per il Sig. Praun mit konzertantem Fagott komponiert hat. (Christoph Praun war der langjährige und damals der beste Bassist der Kaiserlichen Hofmusikkapelle.)

Die mit „1739“ datierten Kompositionen von Francesco Durante:

Salve Regina con VV. Violetta con un tocco di fagotti ... per il Sig. ${ }^{r}$ Praun (Autograph, GB-Bl),

Alma Redemptoris Mater (B+Orch, „dicembre 1739“, A-Wn),

Alma Redemptoris Mater (S+Orch, 1739, A-Wn),

Missa in Palestrina (4 voci e basso, Oktober 1739, mehrere Abschriften).

Durante konnte im Herbst 1739 in Wien von mehreren Musikern (Mitglieder der Kaiserlichen Hofmusikkapelle, Italiener usw.) von einer freien Musiker-Stelle am Preßburger Hof des Fürstprimas Esterházy erfahren haben, er konnte theoretisch auch mit dem ehemaligen Esterházyschen Musiker Joseph Umstatt (1711-1762) zusamengetroffen sein. (Umstatt stammte aus Wien und im Sommer 1739 ist er aus dem Dienst beim Fürstbischof ausgetreten.)

Über den Aufenthalt Durantes in Preßburg wissen wir nicht viel: er war bestimmt der bestbezahlte Musiker am Hof Esterházys - vierteljährlich bekam er $125 \mathrm{Fl}$, d. h. einen ähnlichen Lohn wie die beiden Hofärtzte (F. X. von Mannagetta und C. J. Perbegg von Thalfeld). Durante wohnte wahrscheinlich im Primatialpalais, weil im Februar 1740 für ihn neue Bettwäsche gekauft wurde („Die 29. febr. 17408 fl 54 x pro tela ad linteamina p. Dno Duranda empta.").

Wir wissen auch, dass Durante, wie auch alle andere Italiener am Hof Esterházys, nicht zu besonders „kränkelnden“ Hofbeamten gehörte. Ob Durante länger in Preßburg blieb, wissen wir nicht, die Archivdokumente (sog. Hofmeisterprotokolle) aus den Jahren 1741-1742 fehlen im Primatialarchiv. Es wäre besonders interessant das zu wissen, weil im Juni 1741 in Preßburg die Krönung Maria Theresias zum ungarischen König stattfand. Durante ist aber wahrscheinlich kurz nach der Jahreswende nach Italien zurückgekehrt, weil am 27. 2. 1741 seine erste Frau starb, die um 12 Jahre ältere Orsola de Laurentis („maladetta vecchia“). Es erscheint sehr unwahrscheinlich, dass Durante nicht zum Begräbnis seiner Frau gereist wäre.

Ein halbes Jahr nach F. Durante, im Juli 1740 kamen zwei italienische Kastraten nach Preßburg - Domenico Tasselli und Dario Luca Catani. ${ }^{13}$ Sie kamen allerdings nicht direkt aus Italien, sondern aus Böhmen, bzw. Mähren. Tasselli wirkte in den 1730er Jahren zuerst in Italien, ab 1737 in Brünn und dann in Prag (in der Operngesellschaft von Santo Lapis). ${ }^{14}$ Der aus Pistoia stammente Catani sang 1738-1739 in Holeschau (im Theater

13 Näher über Tasselli und Catani in Mähren vgl. SEHNAL, Jiří. Počátky opery na Moravě. Současný stav vědomostí. In $O$ divadle na Moravě. Acta Universitatis Palackianae Olomucensis, Suppl. 21, Praha 1974, S. 55-77.

14 Der Auswahl aus der Opern, in denen Tasselli mitgewirkt hat:

1730 - Macerata (Teatro di Macerata)

Giovanni Battista Mastini / Pietro Metastasio: L'Ezio

Leonardo Vinci / Pietro Metastasio: Siroe re di Persia 1736 - Venezia (Teatro San Samuele) 
von F. A. Rottal). ${ }^{15}$ Eine kurze „Zwischenstation“ für beide war jedoch wiederum Wien - einen Beweis bringt die Quittung über die Reise G. Calandros nach Wien, der beide Kastraten nach Preßburg brachte:

Super 21 fl Dno Calandro titulo itineralium Expensarum Viennam pro duobus Castratis conducendis datis

Io Giacomo Calandro ho riceuto dal Sig. ${ }^{r}$ Terek uenti uno fiorino (sic!) per mia andata a Vienna a preadere i due Musici Castrati ed in fede di cio ho la presente riceuuta

Presburg 26 Luglio 1740

$$
\text { Io Giacomo Calandro }
$$

Esterházy bemühte sich, seinen Kastraten gute Arbeitsbedingungen zu bieten. Im September 1740 wurden für sie zwei Klavichorde bei dem Preßburger Orgelbauer Václav Janeček/Janíček (1704-1742) angekauft:

Super 12 fl pro duobus clavicordiis ad mandatum Celsitudini Vestrae duobus castratis emptis

Endes unterschriebener bekenne, dass ich von dem gestrengen Herrn Tireck auf die zwey accordierte Clavicordia aus befehl Ihro Hoch Gnädigsten Fürsten mit zwölf Gulden richtig bezahlet wurde

Den 23 September a: 1740

Wenceslaus Janetschek orgel und Instrument Macher zu Presburg

Die Quartalsbelohnung D. Tassellis und D. L. Catanis betrug je 87 Fl 30 Xr. Für Tasselli, der bis Ende 1745 am Esterházyschen Hof blieb, wurden noch im Juni 1744 kostbare

Baldassare Galuppi / Bartolomeo Vitturi: Ergilda

1737 - Treviso (Teatro Dolfin)

Antonio Vivaldi / Antonio Maria Lucchini: Farnace

? / ?: Alcano re degli Unni

1737 - Brünn (Novo Teatro della Taverna)

Matteo Luchini / ?: Teodorico

1738 - Brünn (ibidem)

? / M. Luchini: Argene

M. Luchini / A. Salvi: Arsace

M. Luchini / F. Silvani: Gli veri amici

1739 - Prag (Theater an der Kotzen)

Santo Lapis: L' Egidio (rappresentazione morale)

Santo Lapis / Antonio Salvi: La Ginevra

Mehr über Santo Lapis in JAKUBCOVÁ, Alena und Kol. (Anmekung 9), S. 338-340.

15 Die in Holeschau aufgeführte Opern, in denen D. L. Catani mitwirkte:

1738 Ignaz Holzbauer / Apostolo Zeno: Sesostris König in Egypten

? Giovanni Porta / Francesco Passarini: Amore e Fortuna

1739 Ignaz Holzbauer / Apostolo Zeno: Vologeso re de Parti

Mehr über die Oper in Holeschau vgl. SPÁČILOVÁ, Jana. K repertoáru italské opery v Holešově ve 30. letech 18. století. Opus musicum, 44, 2012, Nr. 6, S. 27-36. 
Stoffe für sein Kleid gekauft. Ob Catani auch 1741-1742 in Preßburg blieb, wissen wir nicht.

Durante, Tasselli, Catani wirkten gelegentlich mit der fürstbischöflichen Kapelle auch bei einem anderen Esterházy, dem Landesrichter (judex curiae) Joseph Esterházy im naheliegenden Schloss Landschütz (heute Bernolákovo, ca. $9 \mathrm{~km}$ von Pressburg), was wir aus den Forschungen von K. Renner wissen: ${ }^{16}$

(Reisen der E. Esterházyschen Kapelle nach Landschütz - Schloss von Joseph Esterházy):

September 1740 - Dem Lorenz gutscher sambt Vorreither, welche die Wellische Musicanten nacher Preßburg geführte, vor 1. Kostgeld geben $34 \mathrm{Kr}$.

Oktober 1740 - Denen fürstlichen Musicis und Castraten, welche das Ambt gesungen, angeschafftermassen geben 25 St. Chremnitzer Ducaten $105 \mathrm{fl}$.

1741 - Einem Landt Kutscher, welcher die Music von Primas regni an Ihro Hochgräflichen Excellenz Geburts-tag nach Lanschitz geführt, bezahlt $3 \mathrm{fl} .30 \mathrm{Kr}$.

Item vor 2 Trompeter von Primas $3 \mathrm{fl} 34 \mathrm{kr}$.

13. 6. 1743 - Denen Musicanten von Primas regni discretion 12 Cremnitzer Ducaten gegeben, thut 50 fl. $24 \mathrm{kr}$.

Vor allem die zweite Summe (105 Fl, bzw. 25 Kremnitzer Ducaten) ist ziemlich hoch.

Der letzte italienische Musiker kam Ende 1743 an den Hof E. Esterházys - Filippo Antonelli (Loreto ca. 1710 - Wien 1760), der Altist (Contraalto) der Kaiserlichen Hofmusikkapelle in Wien. Laut Köchel wurde Antonelli „von seinem stabilierten Dienste von Maria Loretto" 1733 direkt in die Hofkapelle berufen. ${ }^{17}$ Obwohl F. Antonelli kein Sänger von z. B. G. Orsinis Format war, schätzte ihn der Kapellmeister Fux hoch. Er wirke auch als Solist bei einigen Opern- und Oratorienaufführungen mit (z. B. im Oratorium $L a$ Madre de Macabei und in der Oper Sesostri von G. Porsile, beide 1737). Bei der großen Rekonstruktion der Hofkapelle 1741 wurde Antonelli pensioniert, aber er wirkte gelegentlich am Wiener Hof (als sog. supernumerrarius) bis zu seinem Tod. Bei Esterházy hat er ein höheres Gehalt (100 Fl/Quartal) als Tasselli und Catani bekommen und blieb bis Ende 1745 im Dienst.

16 Obwohl sie namentlich nicht genannt sind; siehe näher RENNER, Klára. Az Esterházy grófok pozsonyi udvarának zenei élete a 18. században. Kapcsolat Vivaldival. Magyar Zene, 40, 2002, Nr. 4, S. 443-465: 462. Esterházysche Musiker wirkten z. B. auch in Marianka (Marienthal), im Paulinerkloster nahe von Preßburg, in dem E. Esterházy einst als Vorgesetzter des Paulinerordens wohnte; vgl. SAS, Ágnes. Kirchenmusikzentren und erzbischöfliche Hofkapellen in Ungarn im 18. Jahrhundert. Studia Musicologica Academiae Scientiarum Hungaricae, 46, Fasc. 1/2, Budapest 2005, S. 87 (1745 - „am Nachmittag wurden von den Kastraten italienische Kantaten zu Ehren eines Gastes im Garten aufgeführt").

17 KÖCHEL, Ludwig Ritter von. Die Kaiserliche Hof-Musikkapelle, S. 75, 82, bzw. KÖCHEL, Ludwig Ritter von. Johann Joseph Fux, Hofcompositor und Hofkapellmeister. Wien: Alfred Hölder Verlag 1872, S. 240. 
Damit kommen wir zu den regen Kontakten E. Esterházys mit der Kaiserlichen Hofmusikkapelle in Wien. Außer F. Antonelli wirkten nämlich noch zwei weitere Mitglieder der Wiener Hofkapelle parallel in Preßburg mit: es war der Geiger Johann Otto Rossetter (ca. 1680 - Wien 1754) und sein Sohn Johann Killian Rossetter (Wien 1710 ${ }^{18}$ - ?). Der aus den Niederlanden stammende Vater war schon mindestens seit 1700 in Wien, weil er am 26. Januar $1700 \mathrm{im}$ St. Stephansdom geheiratet hat. ${ }^{19}$ Er hat mehrmals Italien besucht und war ab 1709 einer der besten („Virtuoser Suonatore“) und nach den beiden italienischen Konzertmeistern - N. Matteis d. J. und A. Ragazzi - auch einer der bestbezahlten Geiger der Hofkapelle. In Wien pflegte J. O. Rossetter enge Kontakte mit der Familie Liechtenstein, ${ }^{20}$ er war auch ein angesehener Pädagoge: zu seinen Schülern gehörten z. B. J. G. Orschler, J. Čart, J. Hübner, F. M. und K. Seidels und natürlich sein Sohn Johann Killian. Im Herbst 1727 hat sich J. O. Rossetter um einen Posten des Hofscholars für seinen Sohn bemüht, wobei er außer mit der schweren finanziellen Situation seiner großen Familie auch damit argumentierte, sein Sohn schon als 15-jähriger vor dem Kaiser spielte (er musste also ein großes Talent sein). ${ }^{21}$ Wahrscheinlich deshalb, weil seine Bemühung erfolglos war, kam er im Frühling 1731 zusammen mit seinem Sohn nach Preßburg: am 30.3.1731 wurde dem „Caesareo Musico Rosetj et suo filio“ die ziemlich hohe Summe von 24 Kremnitzer Dukaten (= 124 Fl 80 Xr) noch vor seiner Anstellung bezahlt. Er bekam im August 1731 auch das höchste „Kleidergeld“ (50 Fl) von allen Esterházyschen Musikern. J. O. Rossetter blieb nur bis Anfang 1732 am Hof Esterházys, während sein Sohn fast bis Ende 1733 in Preßburg blieb (mit dem hohen Gehalt von 96 Fl, mit Quartiergeld sogar 103 Fl); dann wurde 1734-1736 der jüngere Rossetter auch Geiger der Kaiserlichen Hofmusikkapelle, allerdings nur als sog. supernumerarius, d. h. ohne ständiges Gehalt, während sein Vater praktisch bis zu seinem Tode in der Wiener Kapelle tätig war. Über das weitere Schicksal Johann Killian Rossetters ist nichts Konkretes bekannt.

Aber auch die gelegentlichen Kontakte E. Esterházys mit den Kaiserlichen Hofmusikern waren sehr intensiv.

18 Laut den Matrikeln aus St. Stephan in Wien (matricula-online.eu/Österreich) wurde am 24. Mai 1710 der Sohn J. O. Rossetters auf dem Namen Joannes Valentinus, im August 1712 wiederum ein Sohn „Joannes Georgius" getauft. Wir sind der Meinung, dass Johann Killian R. mit dem ersten identisch ist, weil sein Taufpater Kilian Reinhardt war, der „Concert-Director“ (Concert-Dispensator) der Kaiserlichen Hofmusikkapelle, während es bei dem zweiten der Organist der Hofkapelle Johann Georg Reinhardt war.

19 ÁGUSTSSON, Jóhannes. Joseph Johann Adam of Liechtenstein, Patron of Vivaldi (Anm. 7), S. 7.

20 Ebenda, S. 7-8.

21 KÖCHEL, Ludwig Ritter von. Johann Joseph Fux, Hofcompositor und Hofkapellmeister. Wien: Alfred Hölder Verlag 1872, S. 415-416. („Johann Otto Rosetter, in die 20 iahr Kay. Violinist mit 9 lebendigen unversorgten Kindern kombt allerunterth. Supplicando ein umb die allerhöchste Gnad, damit sein 16 jähriger Sohn Johannes, welcher vor ein iahr schon die allerhöchste gnad gehabt vor Ihro Kay. May. in den Violin mit Ruhm sich hören lassen, für einen HofScolaren möchte allergst. aufgenommen worden. Obwollen mir nit unbewust ist, dass Ihro Kay. May. nit incliniren Scolaren zu halten; auch ich meiner wenigen Seyts aus gewissen ursachen nit für rathsamb erachte. So glaube ich doch diser Supplicant möchte in ansehung seiner besonderen virtù, 20 iährigen Dienste, grossen armuth und des iünglings trefflichen talento mit durch den Totfahl des gewesten Kay. Hofscolaren Nasotto erledigten 360 fl iährlich allergst. consoliert werden, wo nit mit aufnehmung des Sohns für einen würkhlichen Hofscolaren aufs wenigst dem Vater zu einem beytrag, damit er nit allein seinen Sohn Joannes neben den Violin das Clavir lehren zu lassen in stand gesetzt werde, sondern auch mit seiner zahlreichen Familie leichter leben könne.") 
Viele Zahlungen an die Wiener Musiker sind vor allem aus den ersten Jahren der Existenz der Esterházyschen Kapelle 1726-1729 zu belegen, als der Fürstbischof einige seine Musiker, in erster Linie Blasinstrumentenspieler, nach Wien zur Weiterbildung schickte. Die Lehrer waren immer die besten Hofmusiker, z. B. der Fagottist Johann Jacob Friedrich (er unterrichtete František Dušek):

20. 4. 1727 - Friderico Sihn Viennam eunti numeravit pro Friderico Fagotista Aulico Aureos Cremnicenses 18 $75 \mathrm{Fl} 36 \mathrm{Xr}$

Den $5^{\text {ten }}$ dieses [Dezember 1727] ist auf Gnäd. Befehl frantz tusschek fagottist zu seinem Lehr Meister von Pressburg nach Wienn gegangen $8 \mathrm{Fl} 45 \mathrm{Xr}$

Die zwei ersten Esterházyschen Trompeter - Alexander Agner und Martin Streška - waren wiederum vom „Kayserlichen Trompeter H. Johann“ (wahrscheinlich handelt es sich um Johann Heinisch) zu unterrichten, wie es folgende Zahlungen beweisen:

August 1727 - Tubicini Caesareo jussu Celsissimi ubi nostri assumpti sunt ......50,- Fl

3. 10. 1727 - Lohn Instructori Tubicinum ……….................................... 150,- Fl (!)

1. 12. 1727 - den zwë̈ Trompetern [Alexander Agner und Martin Streška] so mit fürst. Erlaubnuß nach Wien gegangen auf gdiste befehl

$21 \mathrm{Fl} 52 \mathrm{Xr}$

Schickte aus f. gdigsten befehl dem Trompeter Martin [Streška] mit dem Kä̈serl. Kunst Trompeter nach Wien $12 \mathrm{Fl} 39 \mathrm{Xr}$

Nach Preßburg wurden auch der Tenorist Ignaz Finsterbusch (am 15. 3. 1728 wurden „dem Tenoristen von Wienn Mons. Finsterbusch“ 12 Kremnitzer Dukaten ausgezahlt) und der Tänzer und Tanzmeister Tobias Gumpenhuber eingeladen.

Zu den Feierlichkeiten des 50-jährigen Priesterjubiläums Esterházys, die mit großem Pomp begangen wurden, ${ }^{22}$ sind 1738 sogar mehrere „Musici Caesarei“ nach Pressburg gekommen:

„[...] Collegio hocce Canonicorum sexto loco antecedente, progrediebatur, tubis interim, tympanisque laetùm, ac finè intermissienone, sonantibus, $\mathcal{E}$ reliquâ totâ Musicâ partim ex Augustâ Urbe ab ipso Caesare, utpote tubicinibus [...].“

„[...] Musici facere concentum ordinariè solent, in superstructâ ad hoc, theatri ad formam, mole, suis scamnis, rubro panno contectis, distinctâ considebant. Musici autem, Eं qui Viennâa advenerant, $\mathcal{E}{ }^{\circ}$ qui anteà hic existebant, aere Principis annuatim conducti, hoc die hinc inde, ex utroque latere, prope Chorum trabibus, asseribúsque, ne irruere in eos multitudo posset, vallati, cernebantur adstantes [...]“.23

22 Vgl. CHMELINOVÁ, Katarína - RAGAČ, Radoslav. Pätdesiate výročie kňazstva Imricha Esterházyho. Niekolko poznámok k takmer zabudnutej barokovej slávnosti v Bratislave. In Orbis atrium. K jubileu Lubomíra Slavićka. Brno: Masarykova univerzita 2009, S. 521-537. SZELESTEI, László. Esterházy Imre hercegprímás aranymiséje (1738). In (Ed. Orsolya Báthory - Franciska Kónya): Egyház és reprezentáció a régi Magyarországon. Budapest: MTA-PPKE Barokk Irodalom és lelkiség Kutatócsoport 2016, S. 343-359. KAČIC, Ladislav. Kapela Imricha Esteházyho (Anm. 3), S. 212.

23 [DRIESCH, Gerhard Cornelius van den:] Quinquagennalia Sacerdotii sive Annus post acceptum Sacerdotium Quinquagesimus. Reverendissimo et Celsissimo S. R. I. Principe Emerico Esterhazyo [...], s. 1., 1738, S. 10, 13. 
Die kaiserlichen Musiker, zu denen sehr wahrscheinlich u. a. beide Rossetters zählten, aber auch mehrere Hoftrompeter, spielten zusammen mit den Esterházyschen Musikern auch eine abendliche „Serenade“ im Garten des fürstbischöflichen Sommerpalastes:

„[...] Totius diei laetitiam claudebat suavis elegans, ac facta ex artis regulis Musica, quam Serenadam vocant; eam Caesarei, Pontificiique Musici circa vesperam adornabant, quae ad noctis penè concubium producebatur $[\ldots]^{“ 24}$

Laut den Dokumenten wissen wir, dass die Musik zum Fest des 50jährigen Jubiläums der Priesterweihe Esterházys im Jahr 1738 der „Music-Director“ Joseph Umstatt komponierte, ${ }^{25}$ leider ist dort nichts Konkretes spezifiziert. Sehr wahrscheinlich ist, dass damals auch die Musik anderer Esterházyscher Komponisten, wie auch der Mitglieder der Kaiserlichen Hofmusikkapelle gespielt wurde.

Alle Kaiserliche Künstler wurden am Esterházyschen Hof regelmäßig „in Gold“ (d. h. in Kremnitzer Dukaten) bezahlt. Einzig der Hofkapellmeister J. J. Fux wurde „mit Wein bezahlt“; es ist ein Dokument von 24. 6. 1734 überliefert, laut dem an Fux nach Wien „5 Eimer alter [= qualitätsvoller] Ratzersdorfer Wein geschickt wurden:

Das aus gnädigsten befehl Ihro hochfürst. Gnaden vor dem H. Fuchs Kayserlichen Capellen=Meister fünf Eimer alter Ratzerstorfer Wein aus dem herrschaftichen Keller nacher Wien abgeschicket sein worden, wird hier mit bestätiget. Prespurg 24. Jun̈̈ 1734.

Franz Török mppria

Dieses Dokument hängt wahrscheinlich mit einem halbjährigen Aufenthalt J. Umstatts vom Januar bis Ende Juni 1734 in Wien zusammen, wo sich der junge Esterházysche Musiker in Komposition/Kontrapunkt bei Fux weiterbilden könnte.

Man sollte auch die rege Zusammenarbeit des Esterházyschen Hofes mit dem Kaiserlichen Lautenmacher Antoni Posch (1677-1742) in den Jahren 1727-1731 (siehe die Abbildung 4) und mit dem Trompetenmacher Michael Leichamschneider (1676-1751) erwähnen, z. B.:

(Super 32 fl pro Cornuis ad Mandatum Suae Celsitutinis emptis)

Quittung

Als zweÿ und drësig gulden, welche ich endts unterschriebener von Ihro Hochfürstl. Gnaden Herrn Herrm fürsten und Ertzbischofen zu Pressburg, vermög geliferten Jägdhorn sambt einen Verschlag, Richtig empfangen habe, welches Bezeigt meine unterschrift

24 Ebenda, S. 21.

25 KAČIC, Ladislav. Kapela Imricha Esterházyho (Anm. 3), S. 212. 
Wienn d. 24. März Ao $1739 \quad$ Michael Leichambschneider

Kaÿ: Trompeden und Jagdhornmacher

In Wienn, in der Naglergassen mppria

In Wien wurden außerdem regelmäßig Musikalien, sowie die „Fagott- und Oboenröhr“, z. B. bei dem Fagottisten der Kapelle der Kaiserinwittwe Amalie Wilhelmine Johann Georg Körner angekauft: am 5. 10. 1728 wurde z. B. dem „Johann Georg Körner Kä̈. Hoff Musicus für ein tutzet fagott-Röhr" $1 \mathrm{Fl} 48 \mathrm{Xr}$ bezahlt.

Zum Schluss noch einige Bemerkungen zum Musikrepertoire am Hof von Emmerich Esterházy. Es wurde dort bestimmt sehr viel Instrumentalmusik (Sinfonien, Konzerte, Ballmusik, Kammermusik usw.) aufgeführt, aber auch Oratorien, Kantaten und andere Gelegenheitswerke (z. B. zum Namenstag des Fürstprimas), wie natürlich auch Kirchenmusik.

Weil keine Musikaliensammlung, kein Inventar der Preßburger Esterházyschen Kapelle überliefert sind, können wir hypothetisch das Repertoire nur „rekonstruieren“. Es könnte sich aus drei Gruppen von Komponistennamen zusammengesetzt haben:

1/ Die bei E. Esterházy wirkenden Komponisten / komponierenden Musiker:

Johann Matthias Schenauer (? - nach 1745)

Leopold Carl (1698-1744)

Johann Peter Behr (? - ?)

Joseph Umstatt (1711-1762)

Johann Otto Rossetter (ca. 1680-1754)

Louis Détry (1698? - ?)

Francesco Durante (1684-1755)

Carl David von Cronnenstein (1709-1746)

2/ Weitere mit der Kapelle zusammenarbeitende Musiker / Komponisten:

Franz Fauner

Paul Durand ${ }^{26}$

3/ Die bis jetzt bekannten Komponistennamen aus dem Repertoire der Kapelle Joseph Esterházys: ${ }^{27}$

Antonio Vivaldi

Johann Georg Orschler

Ignaz Beyer

26 Über den in Preßburg geborenen bekannten Lautenisten Paul Durand (1712-1769?) und sein Wirken am Esterházyschen Hof siehe näher KAČIC, Ladislav. Neue Angaben zu den Jugendjahren Paul Karl Durands. In Die Laute, Jahrbuch der Deutschen Lautengesellschaft, Nr. XII, Frankfurt am Main 2017, S. 110-119.

27 RENNER, Klára. Az Esterházy grófok pozsonyi udvarának zenei élete (Anm. 15), S. 448-449. 
Pichelberger

Ignaz Jacob Holzbauer

Adalbert Fauner

Besonders interessant ist aus dem Aspekt unseres Themas die dritte Gruppe, u. zw. aufgrund der engen Kontakte beider Preßburger Esterházyschen Kapellen, d. h. jener von Fürstprimas Emmerich Esterházy und jene des Landesrichter (,judex curiae“) Joseph Esterházy. Dessen Sohn Joseph Esterházy d. J. ist im Rahmen der sog. Kavalierstour in Venedig mit A. Vivaldi zusammengetroffen und hat von ihm die Noten gekauft. ${ }^{28}$ Der Hof des Landesrichters hat auch gute Kontake zu Wien gepflegt, wovon der hohe Anteil der in Wien wirkenden Musiker (I. Beyer, J. G. Orschler, I. Holzbauer u. a.) im Repertoire zeugt.

Der Hof des Fürstprimas hatte wiederum Kontakte u. a. mit dem sog. „Vivaldi-Oboisten" Ignazio Sieber (ca. 1680 - nach 1757). Am 16. 1.1740 wurde für ihn das Geld (33 Fl $12 \mathrm{Xr}$ ) in Venedig deponiert. ${ }^{29}$ Italienische Musik spielte gewiss eine wichtige Rolle im Repertoire der Fürstbischöflichen Kapelle; man kann das auch aufgrund der engen Kontakte mit der Kaiserlichen Hofmusikkapelle in Wien beurteilen. In Wien konnte man bestimmt auch die Noten der italienischen Komponisten ankaufen. Außerdem hat z. B. der ehemalige Esterházysche Musiker und Kanzlist F. Syhnn 1729 aus Italien Noten nach Preßburg geschickt. ${ }^{30}$ Leider wissen wir nicht, ob und was F. Durante in Preßburg komponierte. Im Unterschied zu J. M. Schenauer, J. Umstatt, L. Carl usw. sind von ihm keine Quitungen mit Angaben über die kompositorische Arbeit (Bezahlungen für konkrete Kompositionen, sog. Kompositionspapier, bzw. -buch u. ä.) überliefert. Es scheint aber unwahrscheinlich, dass Durante während seines einjährigen Aufenthaltes in Pressburg kein Werk komponierte. Dies bleibt eines der Desiderata im für die Musikgeschichte der Slowakei so wichtigen Themas der Esterházyschen Hofmusik in Preßburg.

Kurz zusammenfassend kann man konstatieren, dass die Wirkung der italienischen Musiker am Hof des Fürstprimas Emmerich Esterházy und die enge Zusammenarbeit mit der Kaiserlichen Hofmusikkapelle in Wien zum hohen Niveau seiner Kapelle entscheidend beigetragen haben.

\section{Beilage}

Die Liste der Mitglieder der Kapelle von Emmerich Esterházy (chronologisch):

Alexander Agner Lautenista, Tubicen

Friedrich Syhnn Cancellista et Musicus $1726-1738(\dagger)$

$1726-1728$

28 Ebenda, S. 446, 448 („Der famose geyger vivaldi hat dem graffen neye musicalien geschenket vom ihme selbst componiret, an dem selbe geschenkt $40 \mathrm{fl}$. $36 \mathrm{kr}$.").

29 KAČIC, Ladislav. Kapela Imricha Esterházyho (Anmerkung 3), S. 216, 252.

30 Ebenda, S. 201. 
Johann Matthias Schenauer Cammer-Musicus, Musicus primarius Antoni Cristiani Cammediener, Hoboista

Hyacinth Pichon Cammer-Musicus

Carl Trautmannsheim Cammerlaquay, später Musicus

Franz Biber Cammerdiener, Musicus

František Dušek Fagotista (Lakei)

Martin Streška Tubicen

Leopold Carl Cammer-Musicus

Johann Peter Behr Musicus

Wenzel Bauer Waldhornist (Lakei)

Johann Balthasar Throner Waldhornist, Laquay

Jacob Henschel Musicus

Hans Michel Blühe Laquay, Waldhornist

Faustina Figuli Hofsängerin

Georg Häring Waldhornist, Cammerlaquay

Johannes Wagner Waldhornist

Joseph Gravinger Laquay simul Musicus

Franz Amend Musicus, Vicebuchhalter

Joseph Umstatt Musicus, Music-Director

Angelo Cavallari Fagotista

Johann Otto Rossetter Caesareus Musicus

Johann Killian Rossetter Musicus

Johann Carl Nabicht Tubicen

Johann Beczwarowsky Waldhornist, Laquay

Jacob Sperlich Laquqay, Musicus, Kopist

Joseph Hilverding Musicus

Jakub Štetina Fagotista (Lakei)

Gottfried Finke Tubicen

Johannes Urban Oboista

Joseph Frosch Waldhornist (Lakei)

Andreas Glasser Waldhornist (Lakei)

Adam Zdanský Musicus Kinskianus

Johann Joseph Stephan Musicus Kinskianus, Bassanista

Michael Meissner Fagotista (Lakei)

Giacomo Calandro Musicus

Louis Détry Fagotista

Paul Schmutz Tubicen

Francesco Durante

Domenico Tasselli Castrat

Dario Luca Catani Castrat

Carl David von Cronnenstein Musicus, Lautenista

Matthias Häck Musicus, Tenorista

Johann Rößler Tubicen
1726-1731, 1736-1745

1726-1739

$1726-1727$

$1726-1737(\dagger)$

$1726-1745$

1726-1727

1727-1731

1727-1744(†)

1727-1729

1727-1736

1727-1729

$1727-1738(\dagger)$

$1728-1739(\dagger)$

$1728-1729$

1728-1745

$1728-1729$

1729

$1730-1738$

1730-1739

1730-1734

1731-1732

1732-1733

$1732-1734(\dagger)$

1732-1745

1733-1745

1734-1745

1734-1745

$1734-1743(\dagger)$

1735-1736

1735-1745

1735-1745

1736-1745

1736-1745

1736-1743(†)

1737-1745

1738-1739

1738-1745

1740

1740-1745

1740-1741?

1743-1745

1743-1745

1743-1745 
Johann Georg Trinkl Musicus

Filippo Antonelli Castrat (Contraalto)

Ignaz Walerski Musicus (Lakei)
(Lakei) 1743

$1744-1745$

$1744-1745$

\section{Bibliography}

ÁGUSTSSON, Jóhannes. "La perfetta cognitione". Francis Stephen of Lorraine, Patron of Vivaldi. Studi vivaldiani, 15 (2015), Venezia: Fondazione Giorgio Cini 2015, S. 119-182.

ÁGUSTSSON, Jóhannes. Joseph Johann Adam of Liechtenstein, Patron of Vivaldi. Studi Vivaldiani, 17 (2017), Venezia: Fondazione Giorgio Cini 2017, S. 3-78.

AMORE, Adriano. Giacomo Calandro, un violinista del '700. Le vie della musica, Jg. 8., 2007, Nr. 46, S. 38-39.

BAHLCKE, Joachim. Ungarischer Episkopat und österreichische Monarchie. Stuttgart: Franz Steiner Verlag 2005.

CHMELINOVÁ, Katarína - RAGAČ, Radoslav. Pätdesiate výročie kňazstva Imricha Esterházyho. Niekol'ko poznámok k takmer zabudnutej barokovej slávnosti v Bratislave. In Orbis atrium. K jubileu Lubomíra Slavícka. Brno: Masarykova univerzita 2009, S. 521-537.

DIETZ, Hanns-Bertold. The Neapolitan School: Francesco Durante (1684-1755) - Aspects of Manuscript Dissemination, Misattributions, and Reception. Music en Perspectiva, 2. Jg., 2009, Nr. 2, S. 7-30.

[DRIESCH, Gerhard Cornelius van den:] Quinquagennalia Sacerdotii sive Annus post acceptum Sacerdotium Quinquagesimus. Reverendissimo et Celsissimo S. R. I. Principe Emerico Esterhazyo [...], s. 1., 1738.

FORGÓ, András. Esterházy Imre és az aulikus politika a 18. század első évtizedeiben. In Ibolya Maczák (Hg.): „Fényes palotákban, édes köfalokban“ (Tanulmányok az Eszterházy családról a források tükrében). Budapest: Szépmívés 2009, S. 65-86.

GALAVICS, Géza. Antonio Galli Bibiena in Ungheria e Austria. Acta Historiae Artium, Jg. 30, 1984, S. 177-263.

GERICKE, Hannelore. Wiener Musikalienhandel von 1700 bis 1778. Graz: Böhlau 1960.

HEGEN, Irene. Die markgräfliche Hofkapelle zu Bayreuth (1661-1769). In LEOPOLD, Silke PELKER, Bärbel (Hg.): Süddeutsche Hofkapellen im 18. Jahrhundert (online Publikation). Heidelberg: Heidelberger Akademie der Wissenschaften 2014, S. 1-54.

JAKUBCOVÁ, Alena und Kol. Starši divadlo v českých zemich do konce 18. století (Osobnosti a díla). Praha: Divadelní ústav - Academia 2007.

JONÁŠOVÁ, Milada. Italské operní árie na svatovítském kůru v Praze - Sehlingova éra. Hudebni věda, 38, 2001, S. 263-301.

KAČIC, Ladislav. Joseph Umstatt (1711-1762) zwischen Barock und Klassik. Bemerkungen zur Stilentwicklung eines mitteleuropäischen Komponisten. In (Ed. Gerold Gruber): Zur Geschichte und Aufführungspraxis der Musik des 16.-18. Jahrhunderts in Mittel- und Osteuropa. Bratislava: Musicforum 2013, S. 201-215.

KAČIC, Ladislav. Kapela Imricha Esterházyho v rokoch 1725-1745. Musicologica slovaca, 5 (31), 2014, S. 189-254.

KAČIC, Ladislav. Neue Angaben zu den Jugendjahren Paul Karl Durands. In Die Laute, Jahrbuch der Deutschen Lautengesellschaft, Nr. XII, Frankfurt am Main 2017, S. 110-119. 
KÖCHEL, Ludwig Ritter von. Die Kaiserliche Hofmusikkapelle in Wien von 1543 bis 1867. Wien: Becksche Universitätsbuchhandlung 1869.

KÖCHEL, Ludwig Ritter von. Johann Joseph Fux, Hofcompositor und Hofkapellmeister. Wien: Alfred Hölder Verlag 1872.

KOLTAI, András. Esterházy Imre. In Esztergomi ersékek. Ed. Márgit Beke. Budapest: Szent Istán társulat 2003, S. 331-338.

KOLTAI, András. Császárhű bökezű remete, Esterházy Imre herczegprímás. In Limes 2005, S. $375-418$.

MÜLLER, Erich H. Angelo und Pietro Mingotti. Ein Beitrag zur Geschichte der Oper im XVIII. Jahrhundert. Dresden: Richard Bertling Verlag 1917.

MÜNSTER, Robert. Die Münchener Hofmusik bis 1800. In LEOPOLD, Silke - PELKER, Bärbel (Hg.) Süddeutsche Hofkapellen im 18. Jahrhundert (online Publikation). Heidelberg: Heidelberger Akademie der Wissenschaften 2014, S. 367-408.

PÖTZL-MALÍKOVÁ, Mária. Juraj Rafael Donner a Bratislava. Bratislava: Tatran 1993.

RENNER, Klára. Az Esterházy grófok pozsonyi udvarának zenei élete a 18. században. Kapcsolat Vivaldival. Magyar Zene, 40, 2002, Nr. 4, S. 443-465.

SAS, Ágnes. Kirchenmusikzentren und erzbischöfliche Hofkapellen in Ungarn im 18. Jahrhundert. Studia Musicologica Academiae Scientiarum Hungaricae, 46, Fasc. 1/2, Budapest 2005, S. 81-98.

SCHMITTH, P. Nicolaus SJ. Archiepiscopi Strigonienes. Pars secunda. Tyrnavie: Typis Acemicis Societatis Jesu 1758.

SEHNAL, Jiří. Počátky opery na Moravě. Současný stav vědomostí. In $O$ divadle na Moravě. Acta Universitatis Palackianae Olomucensi, Suppl. 21, Praha 1974, S. 55-77.

SPÁČILOVÁ, Jana. K repertoáru italské opery v Holešově ve 30. letech 18. století. Opus musicum, 44, 2012, Nr. 6, S. 27-36.

SZELESTEI, László. Esterházy Imre hercegprímás aranymiséje (1738). In (Ed.) Orsolya Báthory

- Franciska Kónya): Egyház és reprezentáció a régi Magyarországon. Budapest: MTA-PPKE Barokk Irodalom és lelkiség Kutatócsoport 2016, S. 343-359.

UMSTATT. Joseph. Concerti per violino, hrsg. von Ladislav Kačic. Bratislava: Vysoká škola múzických umení 2013.

UMSTATT, Joseph. Concerti per flauto traverso, hrsg. von Ladislav Kačic. Bratislava: Vysoká škola múzických umení 2017.

WEINZIERL, Gerhard. Bamberger Hofmusik. Von der Gegenreformation bis zum Beginn des 19. Jahrhunderts. Würzburg: Königshausen und Neumann 2016. 


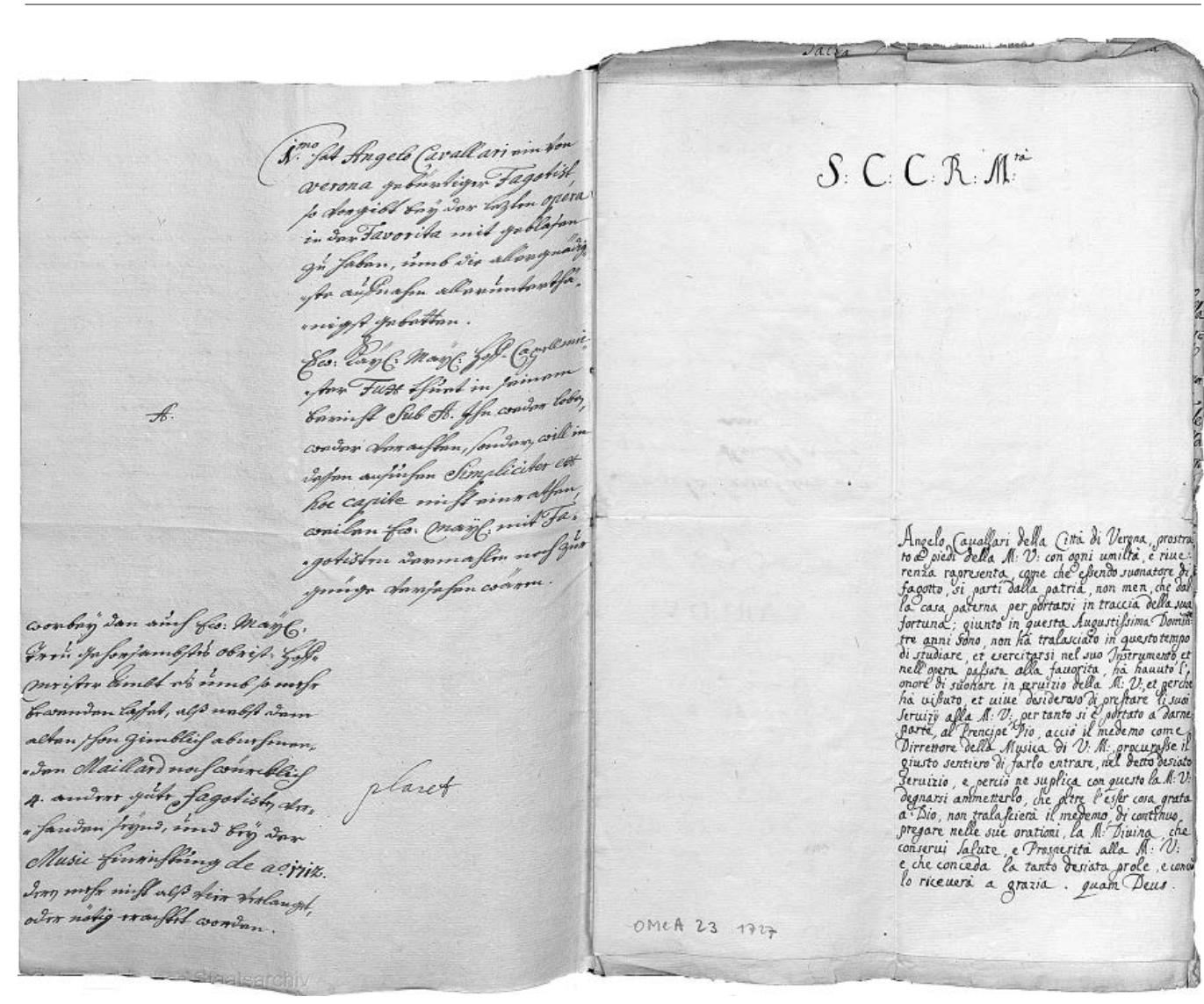

Abbildung 1

Ansuchen von A. Cavallari (1727) um die Fagottisten-Stelle in der Kaiserlichen Hofmusikkapelle (Wien, HHStA, OmeA 23) 


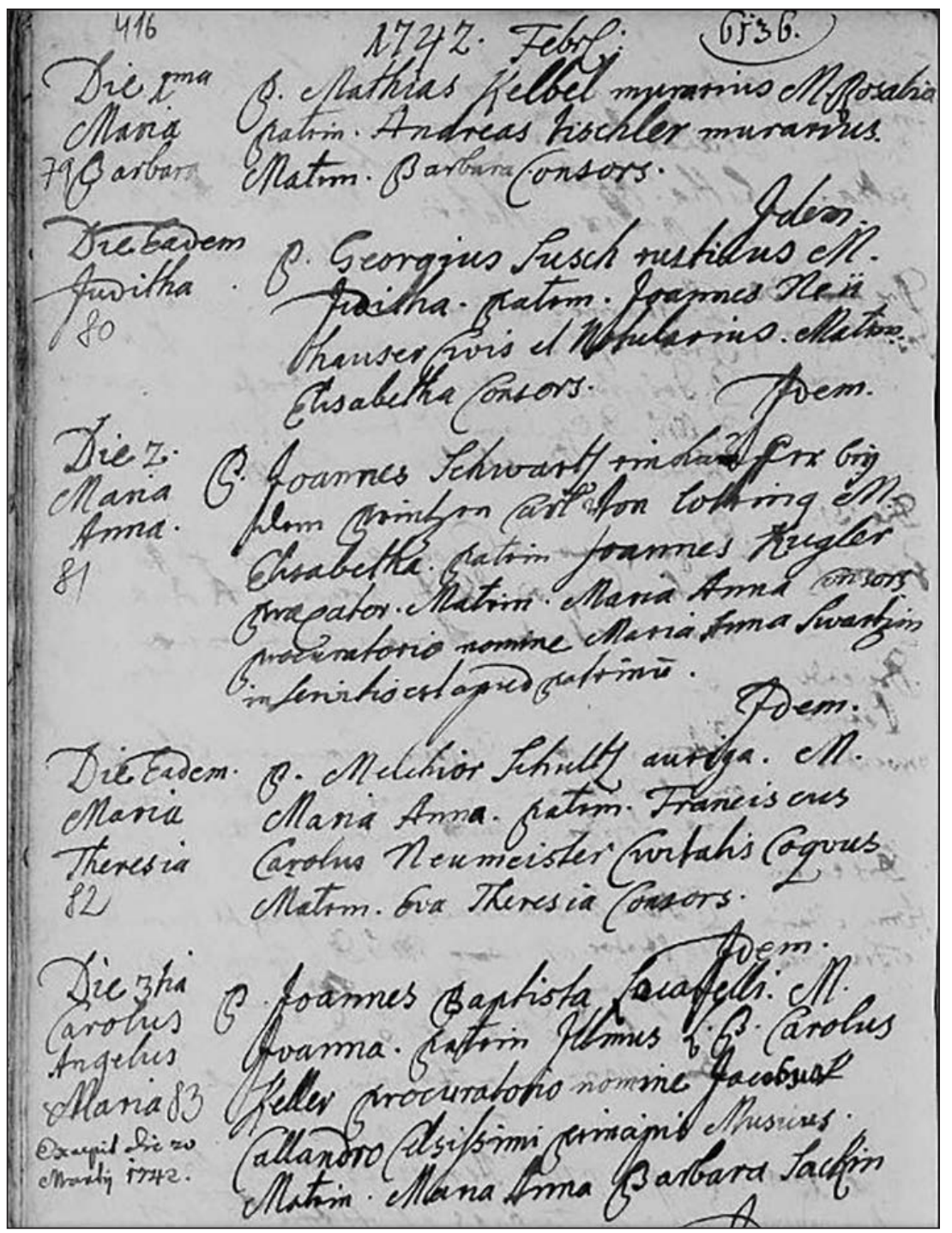

\section{Abbildung 2}

Aufzeichnung über die Taufe (3. 2. 1742) des Sohns von G. B. Locatelli und G. della Stella im Preßburger St. Martins-Dom

(Bratislava, AMB, Zbierka cirkevných matrík) 


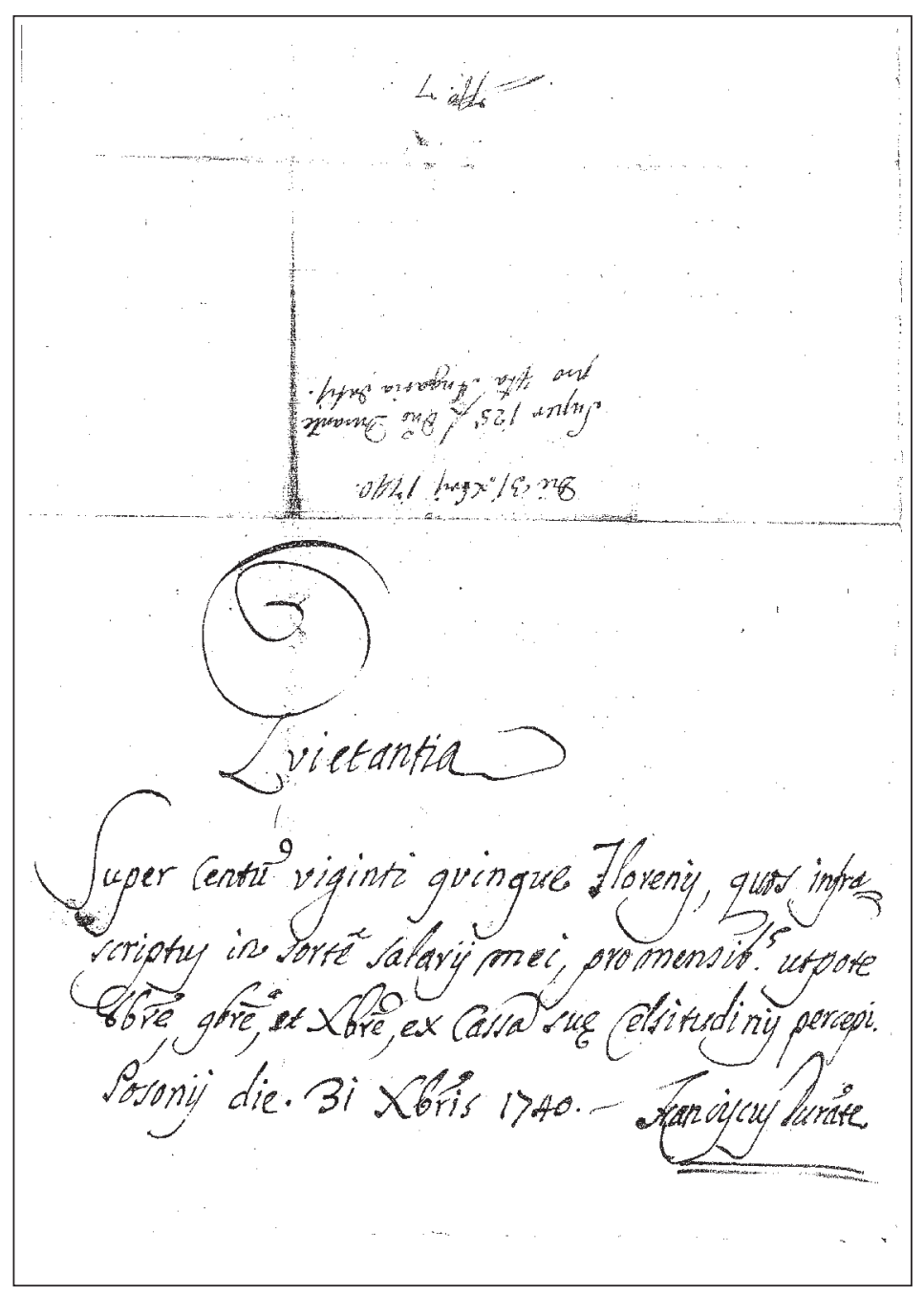

Abbildung 3

Quittung von F. Durante für das 4. Quartal 1740

(Esztergom, Prímási leveltár, Udvarmesteri számádások, Inv. Nr. 19) 


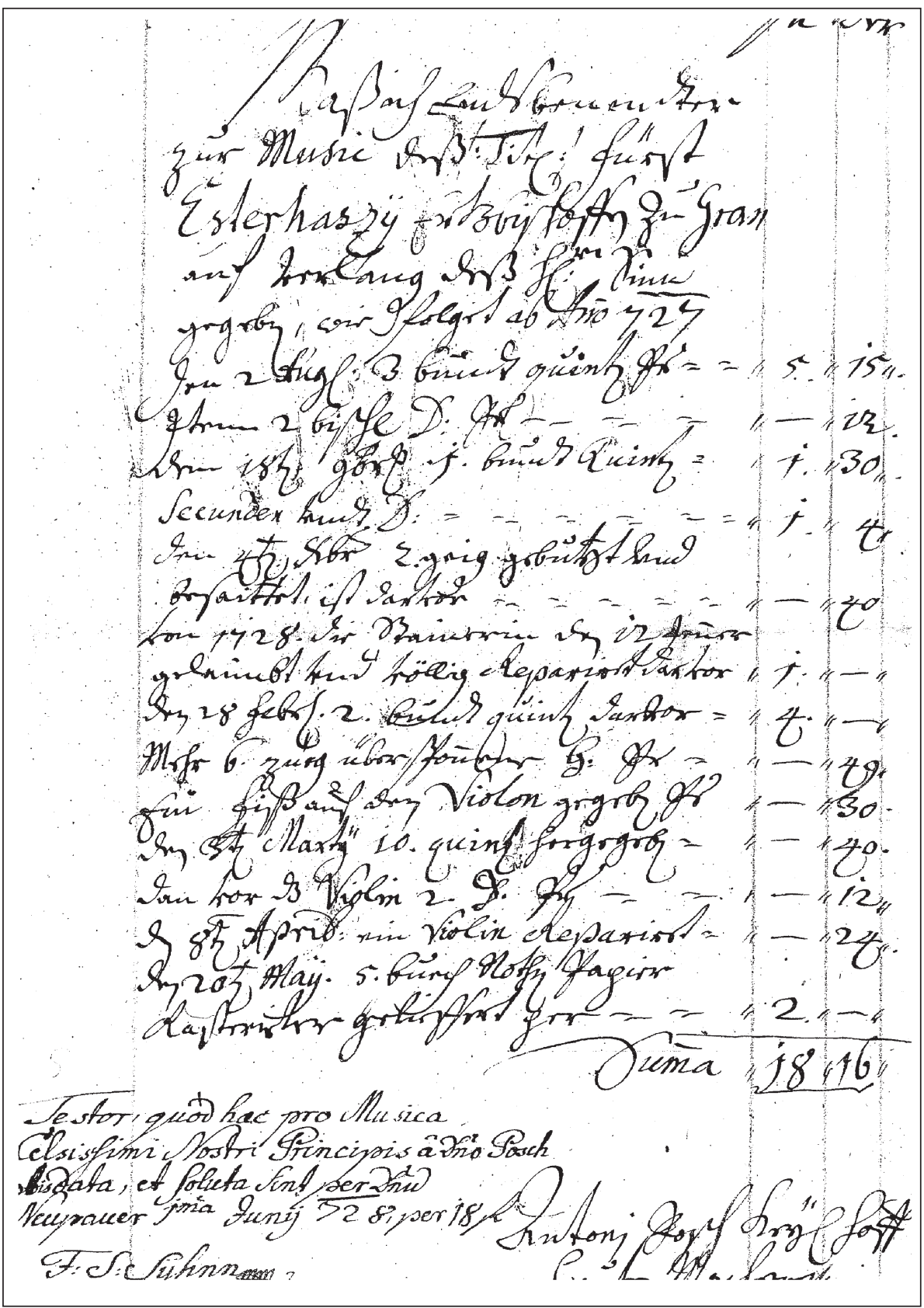

\section{Abbildung 4}

Vertrag mit dem Kaiserlichen Hof-Lautenmacher Antoni Posch (1727/1728)

(Esztergom, Prímási leveltár, Udvarmesteri számádások, Inv. Nr. 8) 


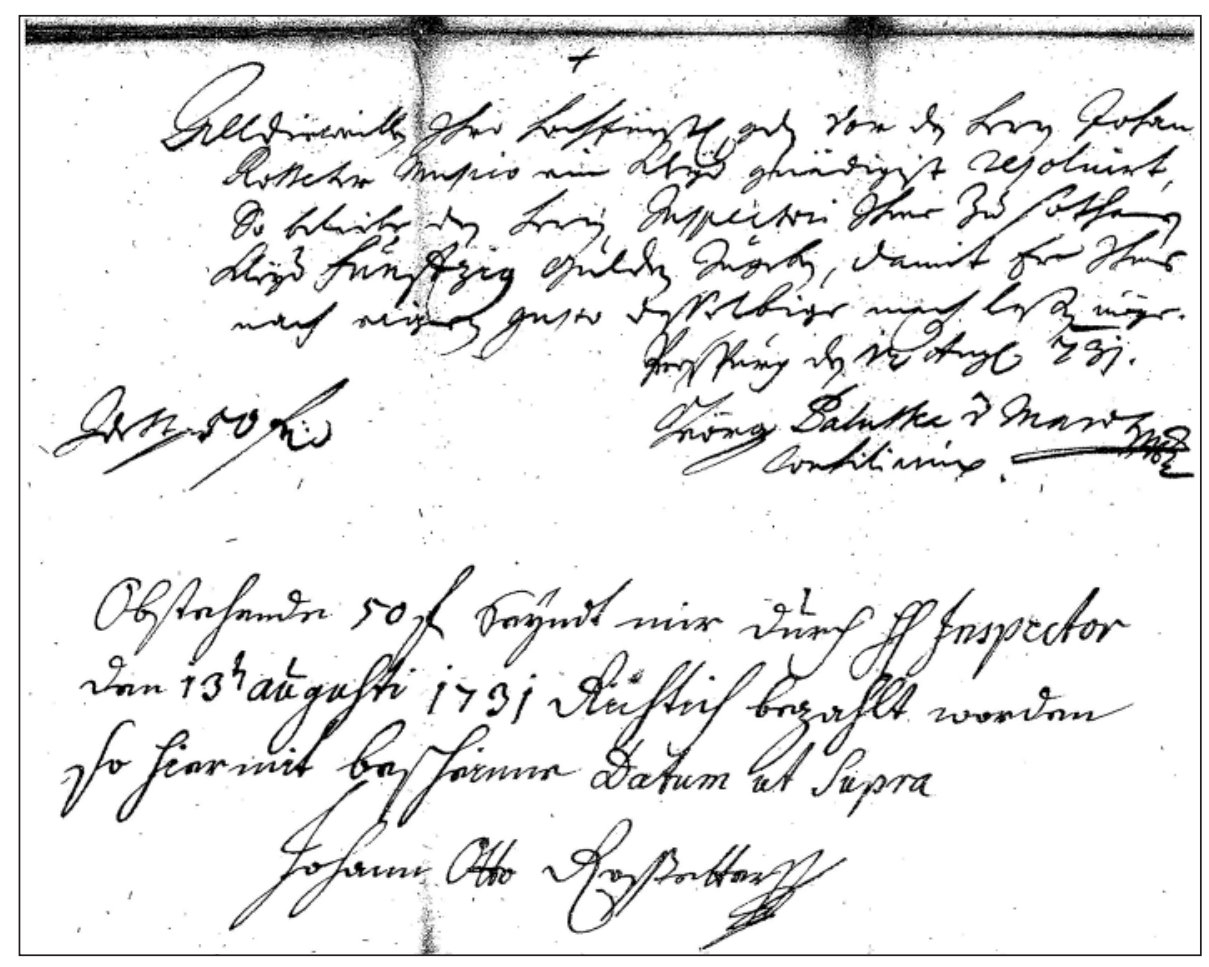

\section{Abbildung 5}

Quittung von Johann Otto Rossetter über das Kleidergeld (1731)

(Esztergom, Prímási leveltár, Udvarmesteri számádások, Inv. Nr. 6) 